\title{
1 Biocompatibility of Polysebacic Anhydride Microparticles with Chondrocytes in 2 Engineered Cartilage
}

3 Sathish Ponnurangam ${ }^{1 \dagger}$, Grace D. O'Connell ${ }^{2}$, Clark T. Hung ${ }^{3}$, and Ponisseril Somasundaran ${ }^{1}$

$4 \quad{ }^{1}$ Earth and Environmental Engineering, 500 W, $120^{\text {th }}$ street, 918 Mudd Columbia University, New York, NY, 10027,

$5 \quad{ }^{2}$ Mechanical Engineering, 5122 Etcheverry Hall, University of California, Berkeley, CA 94720

$6{ }^{3}$ Biomedical engineering, 351 Engineering Terrace, 1210 Amsterdam Avenue, Columbia University, New York, NY, 10027

† Corresponding author, email: sp2522@columbia.edu, Fax: (212) 854-8362

Abstract

One of main challenges in developing clinically relevant engineered cartilage is overcoming limited

11 nutrient diffusion due to progressive elaboration of extracellular matrix at the periphery of the construct.

12 Macro-channels have been used to decrease the nutrient path-length; however, the channels become

13 occluded with matrix within weeks in culture, reducing nutrient diffusion. Alternatively, microparticles can

14 be imbedded throughout the scaffold to provide localized nutrient delivery. In this study, we evaluated

15 biocompatibility of polysebacic anhydride (PSA) polymers and the effectiveness of PSA-based

16 microparticles for short-term delivery of nutrients in engineered cartilage. PSA-based microparticles were

17 biocompatible with juvenile bovine chondrocytes for concentrations up to $2 \mathrm{mg} / \mathrm{mL}$; however, cytotoxicity

18 was observed at $20 \mathrm{mg} / \mathrm{mL}$. Cytotoxicity at high concentrations is likely due to intracellular accumulation

19 of PSA degradation products and resulting lipotoxicity. Cytotoxicity of PSA was partially reversed in the

20 presence of bovine serum albumin. In conclusion, the findings from this study demonstrate concentration-

21 dependent biocompatibility of PSA-based microparticles and potential application as a nutrient delivery

22 vehicle that can be imbedded in scaffolds for tissue engineering.

24 Keywords: Articular cartilage, polysebacic anhydride, microparticles, delivery, lipotoxicity, hydrogel

Introduction

Articular cartilage, the connective tissue of diarthrodial joints, absorbs and distributes large compressive stresses placed on the joint, and enables low friction sliding. Treatment of diseased or 29 damaged articular cartilage is challenging due to its avascular nature and poor self-repair capability [1, 2]. 
Cartilage tissue-engineering techniques that encapsulate chondrocytes within a three-dimensional scaffold have been successful in promoting extracellular matrix deposition in vitro [3-5]. However, nutrient diffusion into the engineered tissue decreases over time due to the elaborated extracellular matrix on scaffold periphery. As a result, compressive mechanical and biochemical properties are lower in the center of the scaffold than the periphery [6]. One approach to overcome limited nutrient diffusion is to decrease the diffusion path length through macro-channels [6] or localized delivery of nutrients throughout the scaffold. Improved nutrient diffusion from macro-channels can be limited due to cells on the channel periphery depositing extracellular matrix into the channel and occluding the channel within a few weeks in culture [6]. In this study, we use nutrients-encapsulated microparticles to deliver nutrients throughout the scaffold.

There are several current and emerging applications that have used biocompatible polymers to fabricate delivery vehicles for drugs, genes, and vaccines [7, 8]. One challenging factor of using delivery vehicles for nutrient distribution is the ability to accurately control the release profile and to provide

14 uniform distribution of delivered nutrients. For example, transforming growth factor- $\beta 3$ (TGF $\beta 3$ ), which is 15 crucial for cartilage growth and development, must be delivered uniformly during the first two weeks in 16 culture [9, 10]. Therefore, an ideal polymeric delivery device should be biocompatible and exhibit a surface erosion profile with fast release kinetics (i.e. order of weeks).

Polyanhydrides are a class of biodegradable and biocompatible polymers that are used for sustained delivery of bioactive agents [11-13]. They have several advantages, including surface erosion properties and tunable degradation rates suitable for short-term release profiles [14, 15]. Previous studies

21 have used polyanhydrides to deliver vaccines, adjuvants, and proteins, and demonstrated that the immunogenicity of these actives is preserved [16-19]. Moreover, polyanhydride-based wafers are approved by the Federal Drug Administration (FDA) for drug delivery to treat brain tumors [20].

Polymer degradation rate is highly dependent on molecular weight, hydrophobicity, $\mathrm{pH}$, crystallinity, porosity, and surface area of the polymeric device [21-27]. Biocompatible polyanhydrides

26 such as poly(sebacic anhydride) (PSA) prepared from sebacic acid can degrade at a faster rate than 27 other biocompatible polymers (eg., poly(esters)) [14, 15, 28]. Copolymerization of PSA with an aromatic 
1 anhydride (e.g., poly(carboxyphenoxy propane-co-sebacic anhydride), P(CPP-SA)) decreases 2 degradation rate, compared to PSA-alone [14]. Polyanhydrides and their degradation products are 3 considered non-cytotoxic [13, 29-35], non-mutagenic [36], non-carcinogenic [13], and are extensively 4 metabolized by rat brain tissue [37, 38]. In particular, $80 \%$ of the sebacic acid was metabolized and 5 eliminated as $\mathrm{CO}_{2}[37,38]$. Other cells, such as aorta epithelial osteoblast-like and smooth muscle cells, 6 also did not show cytotoxic effects due to polyanhydrides [13, 39]. However, recent work suggests that 7 polyanhydrides are biocompatible for $\mathrm{J} 774$ macrophages $\left(0.5-1.25 \times 10^{6} \mathrm{cells} / \mathrm{mL}\right)$ for polymer 8 concentrations up to $2.8 \mathrm{mg} / \mathrm{mL}$, above which cytotoxicity was observed [40].

For nutrient delivery applications in cartilage tissue engineering, in situ release of sufficient bioactive molecules such as vitamin C would require a PSA microparticle concentrations in excess of 50

$11 \mathrm{mg} / \mathrm{mL}$ (see supplementary information). This necessary concentration (i.e. $>50 \mathrm{mg} / \mathrm{mL}$ ) for delivery of an 12 appropriate concentration of nutrients throughout engineered cartilage may affect biocompatibility of 13 polymeric microparticles due to accumulation of degradation by-products. Currently, there is no data in 14 the literature that evaluates the biocompatibility and cytotoxicity of PSA with chondrocytes. Furthermore, 15 there are no studies exploring the potential mechanisms of cytotoxicity or mitigation strategies. Without answering these questions, the utility of polyanhydrides (in particular PSA) as a short-term delivery device

17 in articular cartilage tissue engineering will be very limited.

Therefore, the objectives of this study are to evaluate biocompatibility of PSA polymer with

19 chondrocytes and to evaluate PSA-based microparticles as nutrients delivery device when embedded in

20 agarose hydrogels for cartilage tissue engineering. Specifically, we found PSA polymer to be nontoxic to 21 chondrocytes at or below $2 \mathrm{mg} / \mathrm{mL}$ PSA, above which cytotoxicity was observed. The mechanism of 22 cytotoxicity at higher concentrations is deduced to be due to lipotoxicity because of structural similarities 23 of PSA degradation products with fatty acids. Several mitigation strategies for PSA were tested and 24 bovine serum albumin (BSA) treatment was found to be effective. The results from this study demonstrate 25 potential use of PSA-microparticles with BSA protection as a nutrient or drug delivery vehicle in 26 engineered cartilage. 
Chemicals required for synthesis of polymeric micro-particles were of analysis or higher grade:

3 Sebacic acid (Alfa Aesar, Ward Hill, MA), methylene chloride (Acros Organics, New Jersey - US), 4 poly(vinyl) alcohol (MP Biomedicals, Santa Ana, CA) petroleum ether (Fisher Scientific, New Jersey 5 US), acetic anhydride, ethyl ether, and amitriptyline hydrochloride from (Sigma Aldrich, St. Louis, MO). 6 Aqueous solutions were made from Millipore ${ }^{\mathrm{TM}}$ water (with resistivity of $\sim 18.1 \mathrm{M} \Omega . \mathrm{cm}$ ).

Synthesis and characterization of PSA polymer

The poly(sebacic anhydride) (PSA) was synthesized using sebacic acid as a monomer by melt condensation method [12]. Recrystallized sebacic acid $(5 \mathrm{mg})$ was mixed with $50 \mathrm{~mL}$ of acetic anhydride and stirred for 20 minutes at $40{ }^{\circ} \mathrm{C}$ until a clear solution was obtained. The excess acetic anhydride, after

11 the de-hydration and acetylation of the diacid groups of sebacic acid monomer, was removed by increasing the temperature to $70-120{ }^{\circ} \mathrm{C}$ under vacuum. The precursor sebacic anhydride molecule was 13 polymerized by increasing and maintaining temperature at $150{ }^{\circ} \mathrm{C}$ with constant stirring for 2 hours. The

14 final PSA product was dissolved in a minimum amount of methylene chloride and recrystallized using a large excess of ethyl ether and petroleum ether. The resulting suspension was centrifuged, dried at room

16 temperature under vacuum, and stored at $-20{ }^{\circ} \mathrm{C}$. The chemical identity of the polymer was characterized 17 using nuclear magnetic resonance (NMR) and vibrational spectroscopies which confirmed successful synthesis of the PSA polymer (see supplementary information).

\section{Fabrication of PSA microparticles and encapsulation of bioactive molecules}

Fabrication of PSA microparticles with encapsulated nutrients into was performed using a double 21 emulsion method (water/oil/water) [41]. Methylene chloride was used as an oil phase and poly(vinyl) 22 alcohol (PVA) as an emulsifier at the interface between the external water and oil phases. An evaporative 23 process was used to eliminate methylene chloride leaving behind the polymer and encapsulated 24 bioactives in the internal water phase. Alternatively, bioactive solids were encapsulated by using them as 25 the internal phase (solid/oil/water) instead of their aqueous solution. In particular, $100 \mathrm{mg}$ of PSA was 26 dissolved in the $1 \mathrm{~mL}$ of methylene chloride and the bioactive internal phase, either as aqueous solution 
or solid phase, was emulsified/dispersed by ultra-sonication and homogenization for 60 seconds. A

2 typical internal phase composition is $5-50 \%$ by weight of the encapsulating polymer [41-43]. Therefore,

3 we set the internal phase at $50 \%$ by weight of PSA (i.e. $50 \mu \mathrm{L}$ of aqueous bioactive to $100 \mathrm{mg}$ of PSA) to

4 maximize encapsulation. This water/oil emulsion was added drop-wise to $2 \mathrm{~mL}$ of the external phase

5 consisting of aqueous solution of emulsifier (PVA) at $1 \mathrm{wt} \%$ under continuous sonication and

6 homogenization for 120 seconds. The obtained double emulsion was then diluted with $20 \mathrm{~mL}$ aqueous

7 solution of $0.5 \%$ PVA and stirred vigorously for 4 hours to evaporate the oil-phase (methylene chloride).

8 A dense layer of polymer thus encapsulates the internal bioactive-phase forming microparticles. The

9 encapsulated-microparticles suspension was centrifuged and washed with water three times. The

10 precipitates were lyophilized and stored at $-20{ }^{0} \mathrm{C}$ until use. The bioactive internal phases that are

11 encapsulated include an aqueous solution of L-Ascorbic acid 2-phosphate sesquimagnesium salt hydrate.

12 The microparticles intended for the delivery in the tissue constructs were fabricated under sterile

13 conditions. Both the internal and external water phases in the double emulsion, as well as water in all

14 stages of fabrication procedure, were replaced with Dulbecco's modified essential medium (DMEM)

15 solution.

Scanning electron microscopy (SEM) was used to characterize size and morphology of PSA

17 microparticles. The microparticles were deposited on a carbon substrate and coated with a $2 \mathrm{~nm}$ coating

18 of Au-Pd and SEM images were obtained with a voltage setting of $0.8 \mathrm{kV}$ and current at $20 \mathrm{~mA}$ (4700-

19 Hitachi SEM, Roslyn Heights, NY).

Previous work on release kinetics from nanogel particles demonstrated that the release profile of

21 amitriptyline, a tricyclic anti-depressant drug, was easily monitored by ultraviolet-light absorption (UV-light;

22 wavelength $=239 \mathrm{~nm})[44,45]$. While amitriptyline has limited applications for cartilage tissue engineering

23 applications, ascorbic acid oxidizes easily in the presence of water and dissolved oxygen, making it

24 difficult to evaluate its release kinetics. Therefore, we evaluated the release kinetics of PSA microparticles

25 by encapsulating microparticles with amitriptyline. Amitriptyline (15 mg) was dissolved in a phosphate

26 buffered saline solution (PBS; $150 \mu \mathrm{L}$ ) and was encapsulated in PSA (200 mg) using double emulsion, as

27 described above. Amitriptyline encapsulated PSA microparticles (175 mg) were resuspended in $10 \mathrm{~mL}$ of 
PBS. To measure the release concentration of amitriptyline from PSA microparticles, the suspension was centrifuged (7000 rpm for 5 minutes) and $5 \mathrm{~mL}$ of the supernatant was extracted once a week for three weeks. Fresh PBS $(5 \mathrm{~mL})$ was added at each time point to maintain the total volume. Amitriptyline standards were made in PBS (range $0-0.025 \mathrm{mg} / \mathrm{mL}$ ) and used to determine the amount of amitriptyline released to the supernatant. cocktail (0.5 ng/mL bFGF, $0.5 \mathrm{ng} / \mathrm{mL}$ PDGF, $1.0 \mathrm{ng} / \mathrm{mL}$ TGF $\beta 1)$ [4]. Once cells reached confluence, they were removed from the culture dish by trypsinization.

Dosage-dependent biocompatibility was evaluated by encapsulating L-Ascorbic acid 2-phosphate sesquimagnesium salt hydrate (stabilized-vitamin C) in PSA microparticles. PSA microparticles were 14 incorporated into a $4 \%$ weight/volume $(\mathrm{w} / \mathrm{v})$ agarose solution $\left(40^{\circ} \mathrm{C}\right.$, Sigma) at varying weight/volume 15 percentages $(0.2 \%, 2 \%, 3 \%$, and $10 \%$ by $\mathrm{w} / \mathrm{v} ; 2,20$, and $100 \mathrm{mg} / \mathrm{mL}$, respectively). Passaged cells 16 (concentration of $60 \times 10^{6}$ cells $/ \mathrm{mL}$ ) were encapsulated in the agarose mixture for a final concentration of $1730 \times 10^{6} \mathrm{cell} / \mathrm{mL}$ in $2 \% \mathrm{w} / \mathrm{v}$ agarose with PSA-microparticles. Each experimental group concentration was cast and studied in separate batches and thus had its own control without PSA microparticles.

Cylindrical constructs were cored from the agarose slab (diameter $=4 \mathrm{~mm}$, thickness $=2.34 \mathrm{~mm}$ ) and cultured in chemically defined medium (DMEM with $0.1 \mathrm{uM}$ dexamethasone, $40 \mathrm{mg} / \mathrm{mL}$ L-proline, 50

$21 \mathrm{\mu g} / \mathrm{mL}$ ascorbate 2-phosphate, $100 \mathrm{mg} / \mathrm{mL}$ sodium pyruvate, $1 \mathrm{X}$ ITS+premix, $100 \mathrm{U} / \mathrm{mL}$ penicillin, and $22100 \mathrm{mg} / \mathrm{mL}$ streptomycin and amphotericin B (Invitrogen Co., Carlsbad, CA)). The medium was changed 23 every three days and supplemented with $10 \mathrm{ng} / \mathrm{mL}$ of TGF $\beta 3$ during the first 14 days in culture. Cell 24 viability was assessed using a live/dead cytotoxicity kit (Molecular Probes, Invitrogen), where living cells 25 are stained with calcein-AM and dead cells are stained with ethidium bromide. 
The efficacy of bovine serum albumin (BSA) as mitigation agent for cytotoxicity of PSA polymer

2 (not microparticles) and its degradation products was evaluated. BSA protein was added during the

3 casting process (at $1.1 \% \mathrm{w} / \mathrm{v}$ BSA and $3 \% \mathrm{w} / \mathrm{v}$ PSA polymer) and in the growth medium at $1 \mathrm{mg} / \mathrm{mL}$. BSA

4 was evaluated at 1:2 ratio of BSA to PSA (i.e. 1.1\% w/v BSA and $3 \% \mathrm{w} / \mathrm{v}$ PSA). Experimental groups

5 without BSA but with $3 \%$ w/v PSA or no PSA polymer served as controls.

\section{Mechanical properties}

7 The equilibrium Young's modulus $\left(E_{Y}\right)$ and dynamic modulus $\left(G^{*}\right.$, at $\left.1 \mathrm{~Hz}\right)$ were measured under

8 unconfined compression ( $n=3-7$ per group) on a custom-built mechanical tester. A $10 \%$ strain was

9 applied at a rate of $0.05 \% / s$ and held for 30 minutes. The equilibrium stress was used to calculate the $\mathrm{E}_{\mathrm{Y}}$.

10 Following stress-relaxation, the dynamic modulus was measured by imposing $2 \%$ sinusoidal strain at

11 frequency $1 \mathrm{~Hz}$.

\section{Biochemical properties}

13 Following mechanical testing, the sample's wet weight was obtained and prepared for biochemical 14 analyses. Constructs were lyophilized for $48 \mathrm{~h}$ to obtain the dry weight and digested overnight using 15 Proteinase $\mathrm{K}$ at $56^{\circ}$ C. The agarose was separated by centrifugation as undigested mass and the 16 supernatant was collected for biochemical analysis. Glycosaminoglycan (GAG) content was assessed 17 using 1,9-dimethylmethylene blue (DMMB) dye-binding assay for measuring sulfated-GAGs. The GAG

18 content was quantified using chondroitin-6-sulfate standards and reported as a ratio of constructs' dry 19 weight. The amount of DNA present in the samples was measured using PicoGreen assay.

\section{Statistical Analysis}

21 All reported data are presented as averages with standard deviations. A one-way ANOVA was performed 22 on mechanical and biochemical properties. Once significance was found, a post hoc t-test was performed 23 to compare experimental groups with the control.

\section{Results and Discussion}


Characterization and release kinetics of polysebacic anhydride microparticles.

The PSA microparticles were spherical with sizes ranging from several hundreds of nanometers

3 to few tens of microns (Figure 1). A 50\% efficiency in amitriptyline encapsulation was observed during the

4 fabrication process. The release profile of amitriptyline from the PSA microspheres was linear during the

5 first two weeks (Figure 2). Within 2 weeks, approximately $60 \%$ of the encapsulated amitriptyline was

6 released from the PSA microparticles, after which the concentration of the released drug was too low for

7 detection (Figure 2). The fast release (< two weeks) and linear profile show that PSA-based polymeric

8 microparticles can be utilized as carriers to provide sustained uniform delivery of nutrients.
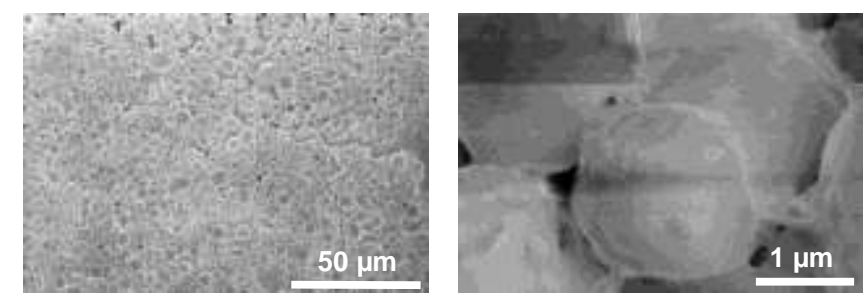

Figure 1 A. Representative scanning electron microscopy (SEM) images of PSA-microparticles. B. High resolution image of a single PSA-microparticle from the same batch.

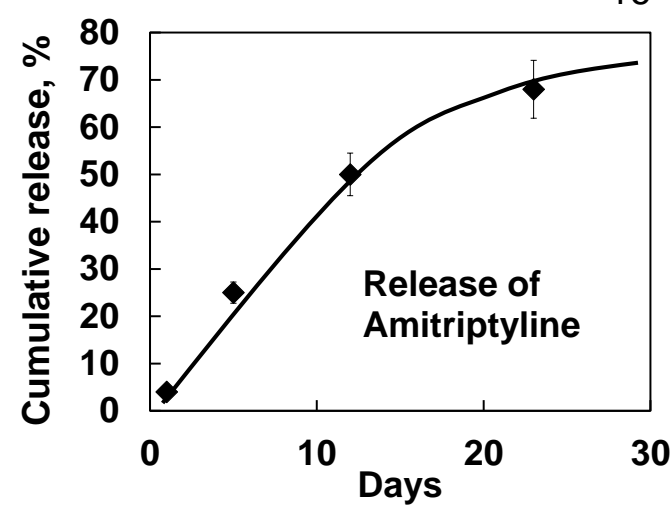

Figure 2 Amitriptyline release profile from PSAmicroparticles (maximum standard deviation, $\mathrm{SD}=9 \%$, for $n=4)$.

\section{Dose dependent cellular viability in the presence of PSA}

The dose-dependence biocompatibility of PSA microparticles with chondrocytes was determined 
However, adding the PSA microparticles at a concentration of $2 \%(20 \mathrm{mg} / \mathrm{mL})$ and $10 \%(100 \mathrm{mg} / \mathrm{mL})$

2 resulted in widespread cell death throughout the construct (Figure 3B \& C). As expected, the drop in cell

3 viability subsequently affected tissue growth and mechanical properties of engineered cartilage (Figure 4;

$4 \quad p<0.01$ versus control). Equilibrium and dynamic moduli of the $0.2 \% \mathrm{w} / \mathrm{v}$ group were not significantly 5 different from the control $(p=0.43)$. Taken together, these results indicate that the non-cytotoxicity of

6 PSA polymeric microparticles is limited to concentrations below $0.2 \% \mathrm{w} / \mathrm{v}(2 \mathrm{mg} / \mathrm{mL})$.

In contrast to the earlier studies on biocompatibility of polyanhydrides [13, 29-35], our results

8 indicate cytotoxicity at higher concentrations of PSA microparticles. This agrees with recent work reported 9 by Adler and coworkers [44], who found cytotoxic effects of polyanhydride copolymer (P(CPP-SA)) at concentrations at or above $2.8 \mathrm{mg} / \mathrm{mL}$. However, the mechanism of sebacic acid polymer cytotoxicity at

11 higher concentrations is not well understood. Moreover, no methods have been proposed to mitigate 12 cytotoxicity of PSA polymer.
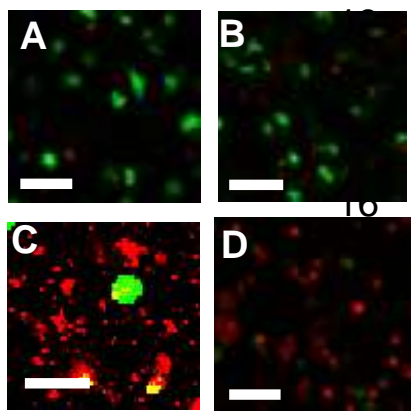

Figure 3 Cell viability of chondrocytes encapsulated in agarose hydrogel with PSA polymer at concentrations of (A) $0 \% \mathrm{w} / \mathrm{v}$ (control group), (B) $0.2 \%$ $\mathrm{w} / \mathrm{v}(2 \mathrm{mg} / \mathrm{mL})(C) 2 \% \mathrm{w} / \mathrm{v}(20 \mathrm{mg} / \mathrm{mL})$ and (D) $10 \% \mathrm{w} / \mathrm{v}(100 \mathrm{mg} / \mathrm{mL})$. Green color indicates living cells and red color indicates dead cells. Bar represents $50 \mu \mathrm{m}$.

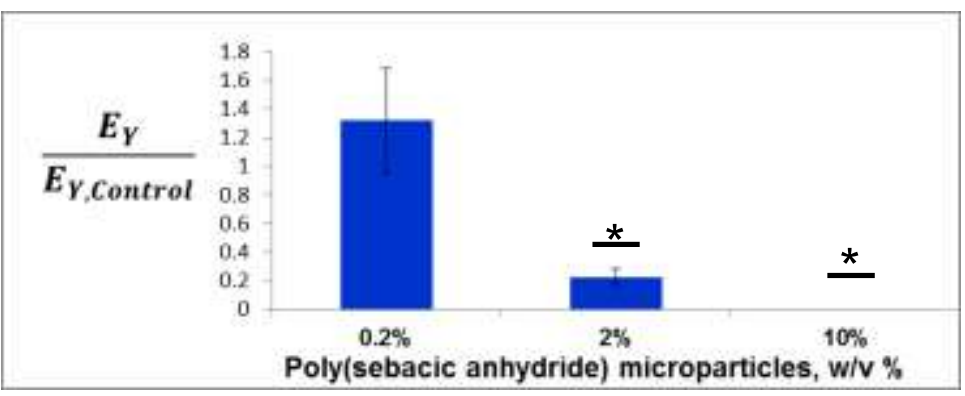

Figure 4 Compressive Young's modulus of engineered cartilage containing PSA-microparticles encapsulated with L-Ascorbic acid 2-phosphate sesquimagnesium salt hydrate. Separate tissue engineering studies were run to evaluate each concentration group; therefore, the values were normalized to the respective control group (i.e. constructs without PSA; represented by the solid black 
experimental group was discontinued after 14 days, due to lack of metabolic activity. $S D=2.5-10 \%, n=3$ per group. ${ }^{*}$ Represents significant differences from the control group $(p<0.01)$.

\section{Mechanisms of the cytotoxicity of PSA microparticles}

Several factors contribute to inflammatory, cytotoxic, or genotoxic effect on cells, including changes in local $\mathrm{pH}$, osmolality, disruption of cytoplasmic membrane, uptake of biomaterials beyond biocompatible limits, $\mathrm{Ca}^{2+}$ levels, lipotoxicity, and induction of reactive oxygen or nitrogen species [46-48].

It is already known that a change in local $\mathrm{pH}$ from the physiological value of 7.2 significantly decreases matrix synthesis from chondrocytes [49]. In particular, the $\mathrm{pH}$ of our growth medium dropped from 7.5 to 6.3 when PSA-microparticles were suspended at $30 \mathrm{mg} / \mathrm{mL}$ for 5 hours. However, increasing the buffer capacity of the culture medium (5mM HEPES buffer) demonstrated that a drop in local pH was not the primary cause for cell death. HEPES buffer stabilized $\mathrm{pH}$ of the culture medium at 7.2, but did not

14 did not improve cell viability in the presence of PSA microparticles (Supplementary Information, Figure 15 S3).

The cytotoxicity of PSA may be due to cellular uptake of degradation products beyond 17 biocompatible limits. The main degradation product of PSA is polysebacic acid, which can be considered 18 a fatty acid-like molecule due to the presence of carboxylic acid groups and long hydrophobic chains. It is 19 well known that excessive intracellular concentrations of alkyl fatty acids can cause abnormal levels of 20 reactive oxygen species $\mathrm{ROS}$ and even result in cellular death $[48,50]$, which is called lipotoxicity. If the 21 chondrocytes ingest the PSA-microparticles or its degradation products, then the alkyl fatty acid-like 22 concentrations inside cells can be expected to increase, causing lipotoxicity. The natural pathway by 23 which cells cope with lipotoxicity is through the intracellular regulation of lipids by serum albumin proteins $24[51,52]$. That is, by maintaining a ratio of serum albumin/lipids of $0.7-2$, cells are able to combat the 25 adverse effects caused by increased concentrations of fatty acids [52].

Thus, we hypothesized that if cytotoxicity was caused by lipotoxicity, the addition of bovine serum 27 albumin (BSA) can mitigate cytotoxicity of PSA degradation products. To test this, BSA was added to the 
agarose hydrogel during casting and the culture medium was supplemented with $1 \mathrm{mg} / \mathrm{mL}$ of BSA

2 throughout the culture period. BSA significantly decreased cytotoxicity from PSA microparticles at a

3 concentration of $3 \% \mathrm{w} / \mathrm{v}$ (Figure 5). At day 30, equilibrium compressive Young's and dynamic moduli of

4 BSA-protected constructs were comparable to the PSA-free control (Figure 5A, and 5B). The biochemical composition, GAG and DNA content (Figure 5C, and 5D day 60), were also similar for these two groups. presents a potential to use PSA microparticles as a delivery vehicle in engineered cartilage constructs.

8 This finding of cytotoxic effects of hydrophobic anhydride polymers is important, since it has been earlier

9 suggested that these polymers, when hydrolyzed to carboxylic acids, can act as nutrients for cells [53, 10 54].
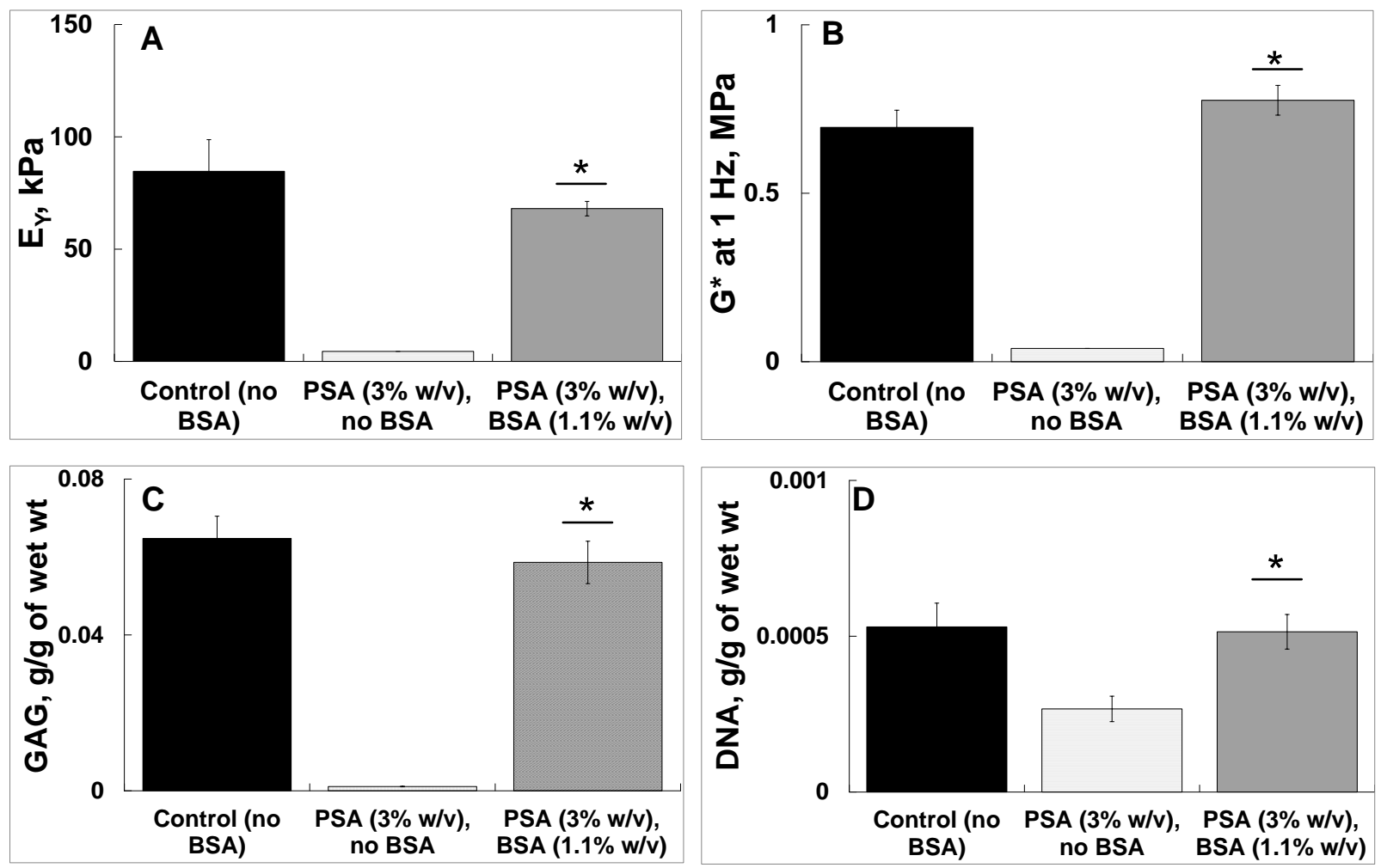
1 Figure 5 Compressive mechanical and biochemical properties of engineered cartilage constructs with 2 BSA protection from PSA degradation byproducts. (A.) Equilibrium Young's modulus ( $\left.E_{Y}\right)$, (B.) Dynamic 3 modulus $\left(\mathrm{G}^{*}\right)$, and (C) GAG content, (D) DNA content. Groups include BSA- and PSA-free control (solid),

4 PSA-polymer without BSA (dots), and PSA-polymer with BSA (slant). The amount of GAG and DNA are 5 reported in grams per gram of wet construct. $S D=2-16 \%, n=4$ per group. ${ }^{*}$ Represents: No significant 6 differences were observed between the control and BSA protected constructs $(p>0.13)$. .

Conclusions

Polysebacic anhydride (PSA) polymer and PSA-based microparticles were evaluated for biocompatibility with chondrocytes and were tested for short-term ( 2 weeks) and sustained release of encapsulated nutrients within an agarose hydrogel for articular cartilage tissue engineering. PSA

13 microparticles were biocompatible with chondrocytes at concentrations below $2 \mathrm{mg} / \mathrm{mL}$ and were found to

14 be cytotoxic at a loading concentration of $20 \mathrm{mg} / \mathrm{mL}$. The mechanism of cytotoxicity is suggested to be

15 due to the intracellular accumulation of PSA or its fatty acid-like hydrolysis products and the resulting

16 lipotoxicity. Bovine serum albumin (BSA) was found to offer the best protection from this adverse effect.

17 Incorporating BSA into the hydrogel and culture system increased the usable PSA-microparticles towards

$183 \% \mathrm{w} / \mathrm{v}(30 \mathrm{mg} / \mathrm{mL})$ within the agarose hydrogel. In this study, we only evaluated one concentration of

19 BSA with PSA polymer. Future work will evaluate whether BSA protection is maintained for higher doses

20 of PSA polymer (e.g. 50-200 mg/mL). In conclusion, the findings reported here show the importance of 21 determining dose dependent effects of PSA on biocompatibility and the potential use of PSA 22 microparticles as a delivery vehicle for cartilage tissue engineering applications.

\section{Acknowledgements}

25 This material is based upon work partially supported by NIH grant AR46568, NSF under Grant 0749461, 26 as well as by NSF and the Environmental Protection Agency under Cooperative Agreement EF 0830117.

27 We acknowledge partial support from Center for Particular and Surfactant Systems (CPaSS) at Columbia 
University and from the Tata Research Development and Design Center, Pune, India. We also thank Dr. Irina Chernyshova for her corrections and suggestions.

\section{References}

[1] G. Vunjak-Novakovic, G. Altman, R. Horan, D.L. Kaplan, Tissue engineering of ligaments, Annu Rev Biomed Eng, 6 (2004) 131-156.

[2] C.M. Hettrich, D. Crawford, S.A. Rodeo, Cartilage repair: third-generation cell-based technologies--basic science, surgical techniques, clinical outcomes, Sports Med Arthrosc, 16 (2008) 230-235.

[3] C.T. Hung, R.L. Mauck, C.C.B. Wang, E.G. Lima, G.A. Ateshian, A paradigm for functional tissue engineering of articular cartilage via applied physiologic deformational loading, Ann. Biomed. Eng., 32 (2004) 35-49.

[4] G.D. O'Connell, J.V. Fong, N. Dunleavy, A. Joffe, G.A. Ateshian, C.T. Hung, Trimethylamine $\mathrm{N}$-oxide as a media supplement for cartilage tissue engineering, Journal of orthopaedic research : official publication of the Orthopaedic Research Society, 30 (2012) 1898-1905. [5] M.D. Buschmann, Y.A. Gluzband, A.J. Grodzinsky, J.H. Kimura, E.B. Hunziker, Chondrocytes in agarose culture synthesize a mechanically functional extracellular-matrix, J. Orth. Res., 10 (1992) 745-758.

[6] L. Bian, S.L. Angione, K.W. Ng, E.G. Lima, D.Y. Williams, D.Q. Mao, G.A. Ateshian, C.T. Hung, Influence of decreasing nutrient path length on the development of engineered cartilage, Osteoarthritis and Cartilage, 17 (2009) 677-685.

[7] M. Vert, Aliphatic polyesters: Great degradable polymers that cannot do everything, Biomacromolecules, 6 (2005) 538-546.

[8] L.S. Nair, C.T. Laurencin, Biodegradable polymers as biomaterials, Progress in Polymer Science, 32 (2007) 762-798.

[9] K.W. Ng, C.J. O'Conor, L.E. Kugler, J.L. Cook, G.A. Ateshian, C.T. Hung, Transient Supplementation of Anabolic Growth Factors Rapidly Stimulates Matrix Synthesis in Engineered Cartilage, Annals of Biomedical Engineering, 39 (2011) 2491-2500.

[10] R.L. Mauck, S.B. Nicoll, S.L. Seyhan, G.A. Ateshian, C.T. Hung, Synergistic action of growth factors and dynamic loading for articular cartilage tissue engineering, Tissue Engineering, 9 (2003) 597-611.

[11] A.G. Braun, C.A. Buckner, D.J. Emerson, B.B. Nichinson, Quantative correspondence between the in vivo and in vitro activity of teratogenic agents, Proceedings of the National Academy of Sciences of the United States of America-Biological Sciences, 79 (1982) 20562060.

[12] A.J. Domb, S. Amselem, J. Shah, M. Maniar, Polyanhydrides - Synthesis and characterization, Advances in Polymer Science, 107 (1993) 93-141.

[13] K.W. Leong, P. Damore, M. Marletta, R. Langer, Bioerodible polyanhydrides as drug-carrier matrices .2. Biocompatibility and chemical reactivity, Journal of Biomedical Materials Research, 20 (1986) 51-64.

[14] A. Göpferich, R. Langer, The influence of microstructure and monomer properties on the erosion mechanism of a class of polyanhydrides, Journal of Polymer Science Part A: Polymer Chemistry, 31 (1993) 2445-2458. 
[15] N. Kumar, R.S. Langer, A.J. Domb, Polyanhydrides: an overview, Advanced Drug Delivery Reviews, 54 (2002) 889-910.

[16] S.K. Lopac, M.P. Torres, J.H. Wilson-Welder, M.J. Wannemuehler, B. Narasimhan, Effect of polymer chemistry and fabrication method on protein release and stability from polyanhydride microspheres, Journal of Biomedical Materials Research Part B: Applied Biomaterials, 91B (2009) 938-947.

[17] M.P. Torres, J.H. Wilson-Welder, S.K. Lopac, Y. Phanse, B. Carrillo-Conde, A.E. RamerTait, B.H. Bellaire, M.J. Wannemuehler, B. Narasimhan, Polyanhydride microparticles enhance dendritic cell antigen presentation and activation, Acta Biomaterialia, 7 (2011) 2857-2864. [18] M.P. Torres, A.S. Determan, G.L. Anderson, S.K. Mallapragada, B. Narasimhan, Amphiphilic polyanhydrides for protein stabilization and release, Biomaterials, 28 (2007) 108116.

[19] M.J. Kipper, J.H. Wilson, M.J. Wannemuehler, B. Narasimhan, Single dose vaccine based on biodegradable polyanhydride microspheres can modulate immune response mechanism, Journal of Biomedical Materials Research Part A, 76A (2006) 798-810.

[20] W. Dang, T. Daviau, H. Brem, Morphological characterization of polyanhydride biodegradable implant gliadel during in vitro and in vivo erosion using scanning electron microscopy, Pharmaceutical Research, 13 (1996) 683-691.

[21] H. Akbari, A. D'Emanuele, D. Attwood, Effect of geometry on the erosion characteristics of polyanhydride matrices, International Journal of Pharmaceutics, 160 (1998) 83-89.

[22] A.J. Domb, R. Nudelman, In-vivo and in-vitro elimination of aliphatic polyanhydrides, Biomaterials, 16 (1995) 319-323.

[23] K.W. Leong, B.C. Brott, R. Langer, Bioerodible polyanhydrides as drug-carrier matrices .1. Characterization, degradation, and release characteristics, Journal of Biomedical Materials Research, 19 (1985) 941-955.

[24] E. Mathiowitz, D. Kline, R. Langer, Morphology of polyanhydride microsphere delivery systems, Scanning Microscopy, 4 (1990) 329-340.

[25] E.S. Park, M. Maniar, J. Shah, Effects of model compounds with varying physicochemical properties on erosion of polyanhydride devices, Journal of Controlled Release, 40 (1996) 111 121.

[26] L. Shieh, J. Tamada, I. Chen, J. Pang, A. Domb, R. Langer, Erosion of a new family of biodegradable polyanhydrides, Journal of Biomedical Materials Research, 28 (1994) 1465-1475. [27] J.A. Tamada, R. Langer, Erosion kinetics of hydrolytically degradable polymers, Proceedings of the National Academy of Sciences of the United States of America, 90 (1993) 552-556.

[28] A.J. Domb, R. Langer, Solid-state and solution stability of poly(anhydrides) and poly(esters), Macromolecules, 22 (1989) 2117-2122.

[29] C. Laurencin, A. Domb, C. Morris, V. Brown, M. Chasin, R. McConnell, N. Lange, R. Langer, Poly(anhydride) administration in high-does in vivo - studies of biocompatibility and toxicology, Journal of Biomedical Materials Research, 24 (1990) 1463-1481.

[30] M.A. Attawia, K.M. Herbert, K.E. Uhrich, R. Langer, C.T. Laurencin, Proliferation, morphology, and protein expression by osteoblasts cultured on poly(anhydride-co-imides), Journal of Biomedical Materials Research, 48 (1999) 322-327.

[31] M.A. Attawia, K.E. Uhrich, E. Botchwey, M. Fan, R. Langer, C.T. Laurencin, Cytotoxicity testing of poly(anhydride-co-imides) for orthopedic applications, Journal of Biomedical Materials Research, 29 (1995) 1233-1240.

[32] A.J. Domb, Synthesis and characterization of biodegradable aromatic anhydride coplymers, Macromolecules, 25 (1992) 12-17.

[33] A.J. Domb, M. Maniar, Absorbable biopolymers derived from dimer fatty acids, Journal of Polymer Science Part a-Polymer Chemistry, 31 (1993) 1275-1285. 
[34] S.E.M. Ibim, K.E. Uhrich, M. Attawia, V.R. Shastri, S.F. El-Amin, R. Bronson, R. Langer, C.T. Laurencin, Preliminary in vivo report on the osteocompatibility of poly(anhydride-co-imides) evaluated in a tibial model, Journal of Biomedical Materials Research, 43 (1998) 374-379. [35] S.M. Ibim, K.E. Uhrich, R. Bronson, S.F. El-Amin, R.S. Langer, C.T. Laurencin, Poly(anhydride-co-imides): in vivo biocompatibility in a rat model, Biomaterials, 19 (1998) 941951.

[36] A.G. Braun, C.A. Buckner, D.J. Emerson, B.B. Nichinson, Quantitative correspondence between the in vivo and in vitro acitivity of teratogenic agents, Proceedings of the National Academy of Sciences of the United States of America-Biological Sciences, 79 (1982) 20562060.

[37] A.J. Domb, M. Rock, C. Perkin, G. Yipchuck, B. Broxup, J.G. Villemure, Excretion of a radiolabeled anticancer biodegradable polymeric implant form the rabbit brain, Biomaterials, 16 (1995) 1069-1072.

[38] A.J. Domb, M. Rock, J. Schwartz, C. Perkin, G. Yipchuk, B. Broxup, J.G. Villemure, Metabolic disposition and elimination studies of a radiolabeled biodegradable polymeric implant in the rat-brain, Biomaterials, 15 (1994) 681-688.

[39] R. Ross, Smooth muscle cell .2. Growth of smooth muscle in culture and formation of elastic fibers, Journal of Cell Biology, 50 (1971) 172-\&.

[40] A.F. Adler, L.K. Petersen, J.H. Wilson, M.P. Torres, J.B. Thorstenson, S.W. Gardner, S.K. Mallapragada, M.J. Wannemuehler, B. Narasimhan, High Throughput Cell-Based Screening of Biodegradable Polyanhydride Libraries, Comb. Chem. High Throughput Screen, 12 (2009) 634645.

[41] A.S. Determan, B.G. Trewyn, V.S.Y. Lin, M. Nilsen-Hamilton, B. Narasimhan, Encapsulation, stabilization, and release of BSA-FITC from polyanhydride microspheres, Journal of Controlled Release, 100 (2004) 97-109.

[42] Y.-S. Lee, P.J. Johnson, P.T. Robbins, R.H. Bridson, Production of nanoparticles-inmicroparticles by a double emulsion method: A comprehensive study, European Journal of Pharmaceutics and Biopharmaceutics, 83 (2013) 168-173. [43] R.L. McCall, R.W. Sirianni, PLGA Nanoparticles Formed by Single- or Double-emulsion with Vitamin E-TPGS, (2013) e51015.

[44] N. Deo, T. Somasundaran, P. Somasundaran, Solution properties of amitriptyline and its partitioning into lipid bilayers, Colloids and Surfaces B: Biointerfaces, 34 (2004) 155-159. [45] P. Somasundaran, F. Liu, S. Chakraborty, C. Gryte Carl, N. Deo, T. Somasundaran, Novel Nanogels for Drug Binding and Delivery, Polymeric Drug Delivery II, American Chemical Society2006, pp. 69-87.

[46] J.P.G. Urban, A.C. Hall, K.A. Gehl, Regulation of matrix synthesis rates by the ionic and osmotic environment of articular chondrocytes, J. Cell. Physiol., 154 (1993) 262-270.

[47] M.H. Wu, J.P.G. Urban, Z.F. Cui, Z. Cui, X. Xu, Effect of extracellular pH on matrix synthesis by chondrocytes in 3D agarose gel, Biotechnology Progress, 23 (2007) 430-434. [48] F.G. Almaguel, J.W. Liu, F.J. Pacheco, C.A. Casiano, M. De Leon, Activation and Reversal of Lipotoxicity in PC12 and Rat Cortical Cells Following Exposure to Palmitic Acid, J. Neurosci. Res., 87 (2009) 1207-1218.

[49] H. Ohshima, J.P.G. Urban, The effect of lactate and pH on proteoglycan and proteinsynthesis rates in the intervertebral-disk, Spine, 17 (1992) 1079-1082.

[50] A. Prasad, M.S. Bloom, D.O. Carpenter, Role of Calcium and ROS in Cell Death Induced by Polyunsaturated Fatty Acids in Murine Thymocytes, J. Cell. Physiol., 225 (2010) 829-836. [51] J.K. Choi, J. Ho, S. Curry, D.H. Qin, R. Bittman, J.A. Hamilton, Interactions of very longchain saturated fatty acids with serum albumin, J. Lipid Res., 43 (2002) 1000-1010.

[52] A.A. Spector, Fatty-acid binding to plasma albumin, J. Lipid Res., 16 (1975) 165-179. [53] D.S. Katti, S. Lakshmi, R. Langer, C.T. Laurencin, Toxicity, biodegradation and elimination of polyanhydrides, Advanced Drug Delivery Reviews, 54 (2002) 933-961. 
1 [54] A.J. Domb, M. Rock, J. Schwartz, C. Perkin, G. Yipchuk, B. Broxup, J.G. Villemure,

2 Metabolic disposition and elimination studies of a radiolabelled biodegradable polymeric implant 3 in the rat brain, Biomaterials, 15 (1994) 681-688. 